\title{
Análisis longitudinal de la profesión de relaciones públicas en España en los últimos cinco años $(2007-2011)^{*}$
}

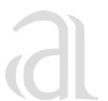

\author{
Cristina Navarro Ruiz* \\ Ángeles Moreno Fernández ${ }^{* *}$ \\ Ansgar Zerfass $^{*+x}$
}

Recibido: 20 de febrero de 2012 Aprobado: 27 de marzo de 2012

\begin{abstract}
Resumen
Desde su primera edición en 2007, los resultados del European Communication Monitor (ECM) han permitido identificar las tendencias en relaciones públicas y la gestión de la comunicación en Europa, describiendo las características principales de los profesionales del sector, así como los instrumentos de comunicación que utilizan, los temas estratégicos, roles ejecutivos o su influencia en las decisiones corporativas, entre otros aspectos. Esta macroencuesta transnacional, que en 2011 contó con la participación de 2.209 profesionales de 43 países, se lleva a cabo en colaboración con académicos de once universidades europeas, la European Public Relations Education and Research Association (EUPRERA) y el apoyo de la European Association of Communication Directors (EACD).

En este estudio se han aislado las preguntas que permiten un análisis longitudinal de las encuestas del ECM entre 2007 y 2011 (Zerfass, Van Ruler, Rogojinaru, Verčič, y Hamrefors, 2007; Zerfass, Moreno, Tench, Verčič, y Verhoeven, 2008, 2009, 2010 y 2011) y los casos de estudio correspondientes a profesionales españoles con el objetivo de ofrecer una visión panorámica sobre la evolución de la profesión en España en el último lustro.
\end{abstract}

Palabras clave: European Communication Monitor, temas estratégicos, canales de comunicación, disciplinas, relaciones públicas en España, roles profesionales, evaluación.

Este artículo de investigación científica forma parte del European Communication Monitor, un proyecto desarrollado por once universidades europeas, entre las que se encuentra la Universidad Rey Juan Carlos en España. Es una investigación organizado por la European Public Relations Education and Research Association (EUPRERA), la European Association of Communication Directors (EACD) y la revista communication Director, patrocinado por Ketchum Pleon, la agencia de comunicación líder en Europa.

* Doctora en Comunicación, de la Universidad Rey Juan Carlos, de Madrid. Actualmente se desempeña como docente de Relaciones Públicas y Periodismo en la Facultad de Ciencias de la Comunicación de la Universidad Rey Juan Carlos de Madrid. Miembro del Grupo de Estudios Avanzados en Comunicación (GEAC) de la Universidad Rey Juan Carlos de Madrid. Correo elecetrónico:crisnavarro@arrakis.es

... Doctora en Comunicación con premio extraordinario por la Universidad Pontifica de Salamanca, España. Actualmente se desempeña como docente de Relaciones Públicas y Comunicación Estratégica en la Universidad Rey Juan Carlos de Madrid, Directora del Título Propio de Experto en Gestión y Evaluación de la Comunicación y el Título Propio de Experto en Planificación y Gestión de Eventos Empresariales. Ha participado como ponente en la Universidad de San Jorge, Universitá di Firenze (IT), Instituto Politécnido de Guarda (PT), Universidad Nacional de Costa Rica (CR) Siracuse University, University of Florida (US), Roskilde University (DN) o University of Jyväskylä (FI). Correo electrónico: mariaangeles.moreno@uric.es

.*** Ansgar Zerfass (Dr., Dr. habil., Universidad de Erlangen-Nuernberg, 1990) es profesor de Gestión de Comunicación en la Universidad de Leipzig, Germany. Antes de unirse a la academia en 2006 ha trabajado en puestos de dirección del gobierno regional, incluyendo la 


\title{
Longitudinal Analysis of Public Relations Practice in Spain during the Last Five Years (2007-2011)
}

\begin{abstract}
Since its first issue in 2007, results of the European Communication Monitor (ECM) have allowed identifying public relations and communication management tendencies in Europe, describing the main characteristics of practitioners of this area as well as communication instruments used, strategic topics, executive roles or its influence on corporate decisions, among other aspects. This transnational macro-survey, where 2,209 professionals from 43 different countries participated in 2011, was conducted in cooperation with academicians from eleven European universities, the European Public Relations Education and Research Association (EUPRERA), and the European Association of Communication Directors (EACD).

In the survey, those questions which allow making a longitudinal analysis of the ECM surveys between 2007 and 2011 (Zerfass, Van Ruler, Rogojinaru, Verčič, y Hamrefors, 2007; Zerfass, Moreno, Tench, Verčič, y Verhoeven, 2008, 2009, 2010 and 2011) and study cases in relation to Spanish professionals were isolated, with the purpose of giving a global overview about the evolution of this practice in Spain during the last five years.
\end{abstract}

Key words: European Communication Monitor; strategic topics; communication channels; disciplines; public relations in Spain; professional roles; evaluation.

responsabilidad de desarrollar industrias creativas en una de las principales regiones alemanas, de preparar programas de educación en profundidad para expertos de comunicación y de liderar un proyecto transnacional de la UE sobre contenido digital. Zerfass es director ejecutivo de EUPRERA, the European Public Relations Education and Research Association (Bruselas), editor del International Journal of Strategic Communication (Routledge Publishers, USA), director del jurado de la International German Public Relations Award (Berlin) y director académico del Corporate Communication Cluster (Vienna). Asimismo, es director científico de la Academic Society for Corporate Leadership and Communication, una iniciativa de compañías globales que apoyan la transferencia de conocimientos en futuros asuntos estratégicos en comunicación corporativa. Zerfass ha recibido diversos premios por su trabajo académico y por sus campañas de comunicación y fue denominado el "investigador de relaciones públicas más innovador en la región germanohablante" en 2010. Es autor y editor de 25 libros y más de 130 artículos y capítulos en diversas lenguas sobre comunicación estratégica e innovación en comunicación. Zerfass es el investigador principal the European Communication Monitor, la principal encuesta anual sobre el estatus y el futuro de la dirección de comunicación en todo el mundo, con datos de más de 40 países. 


\section{Introducción}

En el ámbito de las relaciones públicas, diversos estudios publicados en los últimos años han tratado de analizar la situación y evolución del sector en diferentes países y regiones geográficas (Coombs, Holladay, Hasenauer \& Signitzer, 1994; De Santo \& Moss, 2004; Jang, 1997; J. Grunig, L. Grunig, Sriramesh, Huang \& Lyra, 1995; Nessmann, 1995; Sriramesh \& Kim, 1999; Moreno, Molleda y Suárez, 2006; Moreno, Verhoeven, Tench y Zerfass, 2010; O'Connor \& Muzi, 2004; Swerling, Gregory, Schuh, Goff, Gould, Gu, Palmer y Mchargue, 2008; Swerling, Mitroff, Hall, King, Benson \& O'Boyle, 2005; Swerling, Mitroff, Hall, King, Zeltzer E Waldman, 2004; Swerling, Mitroff, Floto, Hall \& Bishop, 2003; Verhoeven E Aarts, 2010; Zerfass et al., 2008, 2009, 2010, 2011). Estos trabajos han ayudado a avanzar en el conocimiento de la profesión, ilustrar su práctica en todo el mundo y a conducir el debate sobre los principios genéricos que rigen las relaciones públicas (Culberstson \& Chen, 1996; Lim, Goh, E Sriramesh, 2005; Sriramesh E Vercic, 2003; Verčič, Grunig \& Grunig, 1996; Kent $E$ Taylor, 2007; Tilson $\mathcal{E}$ Alozie, 2004). La mayoría de estas investigaciones parten de la base de que las organizaciones, tanto privadas como públicas, necesitan de una función específica al más alto nivel directivo que, además de iniciar y dirigir la comunicación, les otorgue legitimidad ante sus públicos (Beurer-Züllig, Fieseler, E Meckel 2009).

En el caso de España, la producción científica no despegó hasta la llegada de la democracia, superando lentamente el retraso histórico con respecto a Estados Unidos y al resto de Europa en lo referente al estudio y la práctica de la disciplina que acarreó la dictadura franquista (1939-1975). A lo largo de las últimas dos décadas, los investigadores procedentes del mundo académico han abordado el desempeño de las relaciones públicas y su evolución desde diversos ángulos (Arceo, J. L., 2003, 2004; Castillo, 2004a; Fernández y Huertas, 2005; Matilla y Marca, 2001; Moreno, 2004; Míguez, 2007; Noguero, 2004; Xifra, 2008, Tilson y Saura, 2003), centrándose especialmente en el papel de los profesionales dentro de las organizaciones, las disciplinas y canales de comunicación que utilizan, su formación y capacidad de liderazgo, entre otros aspectos.

La literatura científica retrata una disciplina joven, con una trayectoria de poco más de medio siglo y que empezó a dar sus primeros pasos en Barcelona de la mano de Joaquín Maestre en el año 1955 (Noguero, 2004: 337). Su incorporación a los planes de estudio de las universidades no se produjo hasta 1974 y, aunque la primera asociación profesional nació en Madrid en 1961, hubo que esperar hasta 1998 para asistir en Cataluña a la creación un Colegio Oficial de Publicistas y Relaciones Públicas de España.

Sin embargo, según Gutiérrez-García (2010), a pesar de estas importantes contribuciones académicas, la investigación empírica sobre la situación del sector ha sido liderada principalmente por las dos asociaciones profesionales mayoritarias de ámbito nacional: la Asociación de Directivos de Comunicación (Dircom, 2000, 2005, 2008, 2010) y la Asociación de Consultores en Relaciones Públicas y Comunicación (Adecec, 2002, 2004, 2008). En su último estudio, Dircom (2010) presenta un sector en auge, donde los profesionales se ocupan cada vez más de definir las estrategias, gestionar los intangibles y formar parte de la alta dirección de la empresa. De hecho, comparando los resultados con investigaciones anteriores, se detecta un aumento del número de grandes organizaciones que cuentan con un departamento de comunicación específico, pasando del 75,4\% en el año 2000, hasta el 91,3\% en 2010.

Paralelamente, una iniciativa de Euprera (European Public Relations Education and Research Association) comenzó en 2007, con el apoyo de la European Association of Communication Directors (EACD) a identificar los retos actuales y desarrollos futuros de las Relaciones Públicas en Europa, describiendo también la situación profe- 
sional en España. Tras cinco ediciones anuales, el European Communication Monitor (ECM) se ha convertido en la fuente que presenta un análisis más extenso sobre la profesión de gestión de comunicación y relaciones públicas en Europa, ofreciendo al mismo tiempo una interesante radiografía de la situación particular de cada país.

Los datos de España procedentes del ECM (Moreno, Zerfass, Navarro, 2010, 2011 ) muestran un sector afectado por los recortes presupuestarios y a unos profesionales satisfechos con su trabajo, aunque descontentos con el salario y las dificultades que encuentran para conciliar la vida laboral y familiar. La construcción de intangibles aparece como la principal contribución a los objetivos organizacionales y se aprecia que su influencia en las organizaciones es cada vez mayor, ya que el $83 \%$ de los encuestados asegura que los directivos toman en cuenta sus recomendaciones. En cualquier caso, al margen de la disminución de recursos, los profesionales españoles afrontan la actual crisis económica desde una perspectiva proactiva, recurriendo a distintos tipos de estrategias: centrarse en los temas y públicos más relevantes; enfrentarse a una demanda creciente para evaluar sus resultados e invertir en nuevos instrumentos y herramientas. En cuanto a las principales disciplinas dentro de la profesión, se sitúa a la cabeza la comunicación corporativa y, respecto a los canales de comunicación, siguen ocupando una posición dominante los medios impresos, seguidos de los audiovisuales y las herramientas online, cada vez más importantes en la planificación estratégica de las relaciones públicas.

A partir de los datos extraídos de esta encuesta transnacional, este artículo aborda el desarrollo de la profesión en España durante el último lustro: hace un seguimiento de las disciplinas, los instrumentos de comunicación y los temas estratégicos que afectan al sector; identifica las características individuales de los profesionales y evalúa las tendencias en los temas relacionados con la comunicación interactiva. Se concretan así los siguientes objetivos de investigación:

(1) Analizar la evolución de las disciplinas, los instrumentos de comunicación y los temas estratégicos.

(2) Conocer el desarrollo de las tendencias en la comunicación interactiva.

(3) Analizar la evolución del perfil sociodemográfico de los profesionales de las relaciones públicas en España en los últimos cinco años.

\section{Metodología}

Para alcanzar estos objetivos se ha llevado a cabo un análisis longitudinal de los datos relativos a España recogidos en las cinco ediciones del European Communication Monitor (ECM). La información se ha obtenido a través de encuestas, integradas por varias secciones con preguntas cerradas, que se han distribuido vía e-mail a más de 30.000 profesionales europeos de relaciones públicas y gestión de la comunicación en activo, que trabajan en departamentos, consultorías y agencias, organizaciones sin ánimo de lucro y oficinas gubernamentales.

Desde su inicio en 2007, este estudio, conducido por un grupo de profesores de once universidades europeas, ha ido incorporando nuevos países a la muestra y ampliando el número total de partipantes. De hecho, de los 1.087 profesionales pertenecientes a 22 países que contestaron la encuesta en la primera edición, se ha pasado a un total de 2.209 cuestionarios de 43 países en el año 2011. Aunque los autores han ido introduciendo algunas variaciones en el diseño de los cuestionarios aplicados a lo largo de los cinco años, existe una serie de temas básicos que permiten realizar un estudio longitudinal para comprobar los procesos de cambio directamente asociados al paso del tiempo. Estos aspectos son concretamente: temas estratégicos, salarios, disciplinas y medios. 
Las respuestas del cuestionario se procesaron con SPSS y se aplicó a los datos un análisis estadístico descriptivo y analítico. Los resultados y la correlación de variables fueron estadísticamente probados con chi-cuadrado, coeficiente de correlación de Sperman (rho) y análisis de varianza (ANOVA) con test Post Hoc Scheffe, considerando apropiada una significación apropiada ( $\mathrm{p} \leq 0.05)$.

\section{Resultados}

De acuerdo con los objetivos planteados, se exponen en este artículo los resultados más relevantes que se derivan del análisis de datos.

\section{Seguimiento de los temas estratégicos}

Respecto a los temas estratégicos relacionados con el desempeño de la profesión, el estudio longitudinal demuestra que el peso de la 'construcción y el mantenimiento de la confianza' han disminuido en estos últimos cinco años $(-10,1 \%)$, mientras que, como era de esperar, aumenta considerablemente la importancia de 'manejar la evolución digital y la web social', que pasa de un 33,3\% en 2007, a un 60,9\% en 2011. Este incremento está relacionado con la reciente incorporación a la comunicación organizacional de nuevas herramientas y canales online, especialmente con la emergencia de las plataformas interactivas y colaborativas 2.0. De hecho, como se puede observar en la gráfica 1, este tema es considerado el más importante de todos los que afectan al campo de las relaciones públicas.

También se aprecia un aumento, aunque con algunos altibajos, de la necesidad de 'vincular las estrategias de comunicación y los negocios' $(+41,3 \%)$, al tiempo que se reduce en diez puntos la obligación de 'manejar la demanda de nueva transparencia y las audiencias activas'. Crece también la nececidad de establecer nuevos métodos para evaluar la comunicación $(+12,3 \%)$, un dato que ratifica las voluntad de las organizaciones de exigir resultados y la voluntad de los profesionales de rendir cuentas sobre la eficacia de las relaciones públicas. La 'globalización de la comunicación' y los retos que lleva aparejados es otro de los temas estratégicos en auge, según los trabajadores encuestados.

\section{Gráfica 1. Evolución de los temas estratégicos}

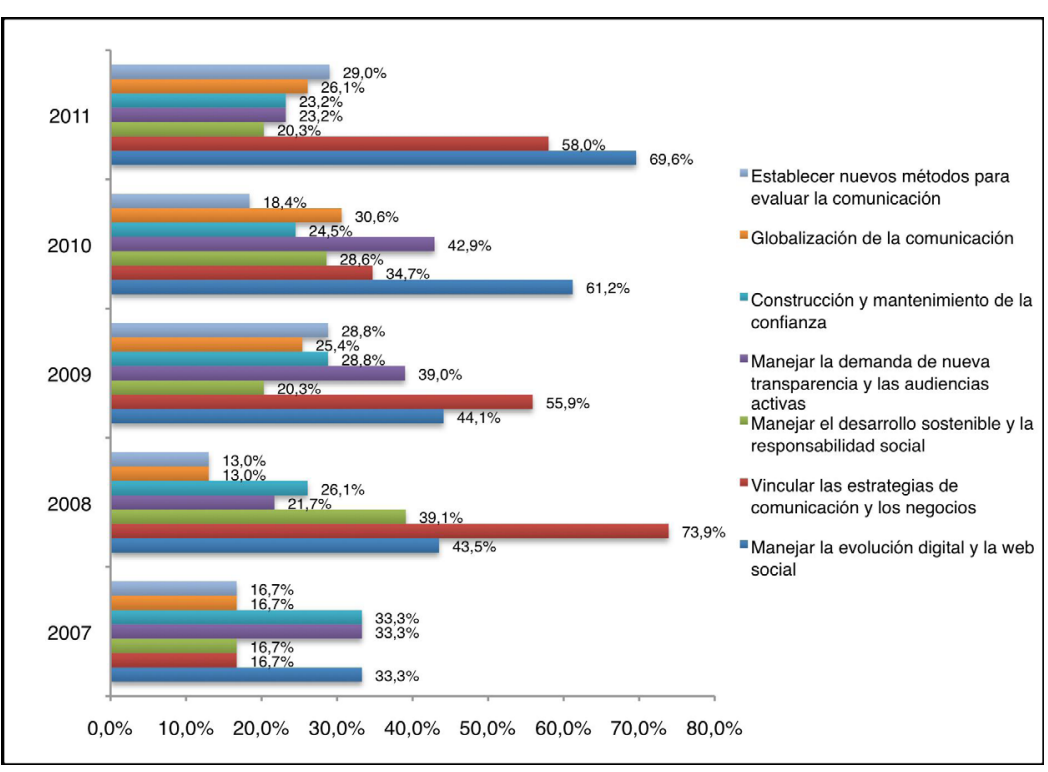

Fuente: elaboración propia. 


\section{Evolución de las disciplinas de relaciones públicas}

La comunicación corporativa -comunicación centrada en la organización en su conjunto- se ha mantenido durante los últimos cinco años como la disciplina más importante para el sector de las relaciones públicas, incrementando incluso su importancia en casi 20 puntos respecto a 2007. De hecho, todas las especialidades analizadas han crecido, excepto la comunicación de crisis y los asuntos públicos (Lob6ying), que bajan un 6\%.

En cuanto a la relevancia que otorgan los profesionales encuestados a cada una de las disciplinas, tras la comunicación corporativa se sitúa la comunicación de marketing/marca y consumidor, seguida por la comunicación de crisis y la responsabilidad social corporativa, que es la que experimenta un mayor crecimiento en los últimos cinco años. En último lugar aparece la comunicación interna y los asuntos públicos, que el 44,1\% de los profesionales consideraba importante en 2011. (Gráfica 2)

\section{Evolución de los canales de comunicación}

Respecto a los canales de comunicación más relevantes para la disciplina, los medios impresos mantienen una cómoda hegemonía, a pesar de la pujanza de las distintas formas de comunicación online y de la incertidumbre que existe sobre el futuro de los periódicos y revistas tradicionales. De hecho, en el último año los profesionales españoles señalan un crecimiento de las relaciones con los medios de comunicación impresos de un 9 por ciento, superando incluso las cifras registradas en 2007. El crecimiento se extiende al resto de los canales, de forma especialmente significativa en el caso de la comunicación online $(+45,1)$ y las relaciones con los medios online $(+37,1)$. La única excepción se encuentra en la comunicación no verbal, que baja del 47,8\% en 2008 al 32,9\% en 2011.

Contrariamente a lo que se podría esperar, teniendo en cuenta la rápida incursión de las herramientas interactivas y colaborativas en la

Gráfica 2. Evolución de las disciplinas

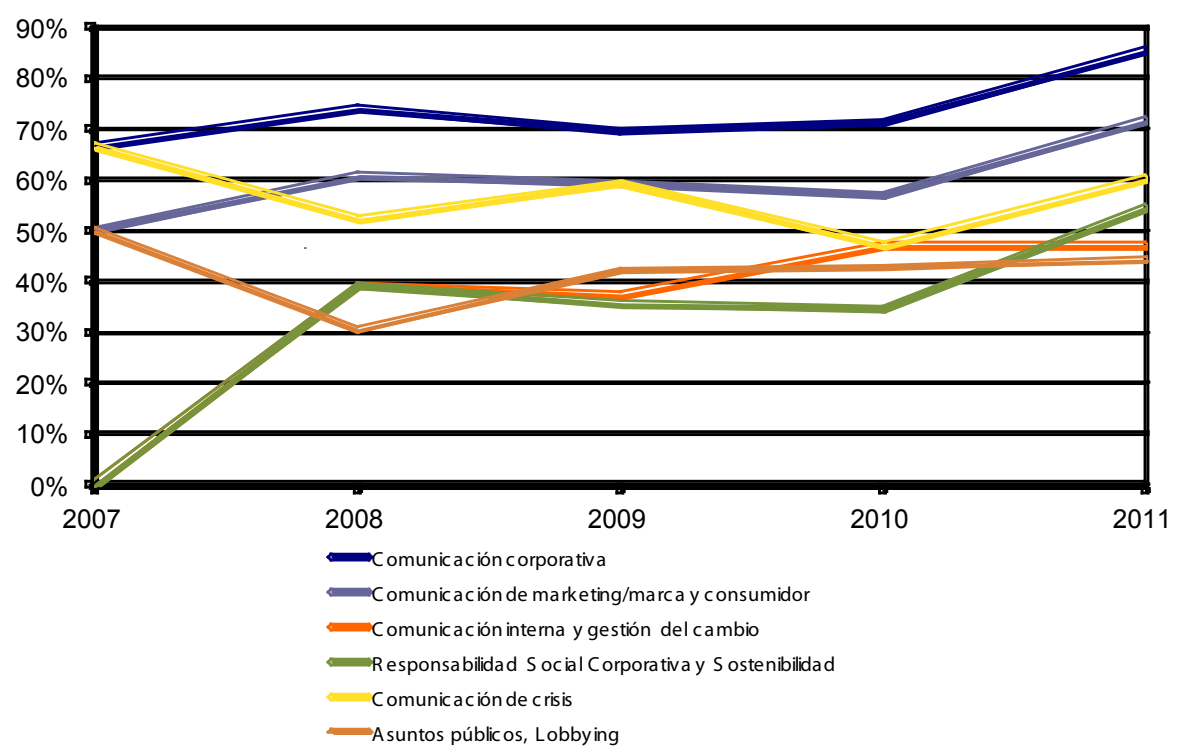

Fuente: elaboración propia. 
comunicación organizacional, solo el 38,8\% de los encuestados considera importantes los Social Media para el desarrollo de su labor. Las relaciones con las emisoras de radio y televisión también incrementan su importancia, a pesar del pequeño bache de 2010, manteniendo el segundo lugar tras los medios impresos. Sin embargo, si la tendencia ascendente de la comunicación online continúa es muy probable que el próximo año desbanque del segundo puesto a los medios audiovisuales tradicionales. (Gráfica 3)

A pesar de la solidez que están demostrando los medios impresos, la gráfica 4 pone de manifies- to el constante crecimiento de los canales de comunicación online durante los últimos cinco años, una dinámica que está influyendo decisivamente en la evolución de la disciplina y transformando radicalmente el paradigma de la comunicación dentro de las organizaciones. En concreto, el uso de los Social Media experimenta una subida cercana a los 30 puntos, al igual que las relaciones con los medios online. Este rápido incremento constituye uno de los principales retos a los que se enfrentan en la actualidad los profesionales de las relaciones públicas y que, sin duda, está influyendo en la planificación y las estrategias comunicativas de las organizaciones. (Gráfica 4)

Gráfica 3. Evolución de los canales de comunicación

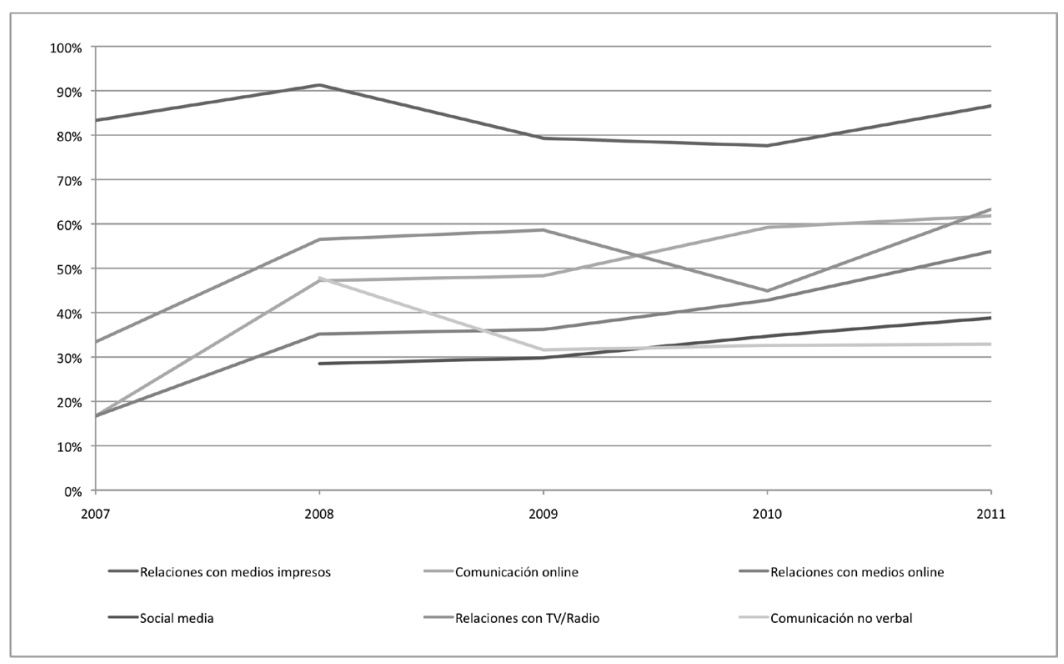

Fuente: elaboración propia.

Gráfica 4. Evolución de la comunicación online

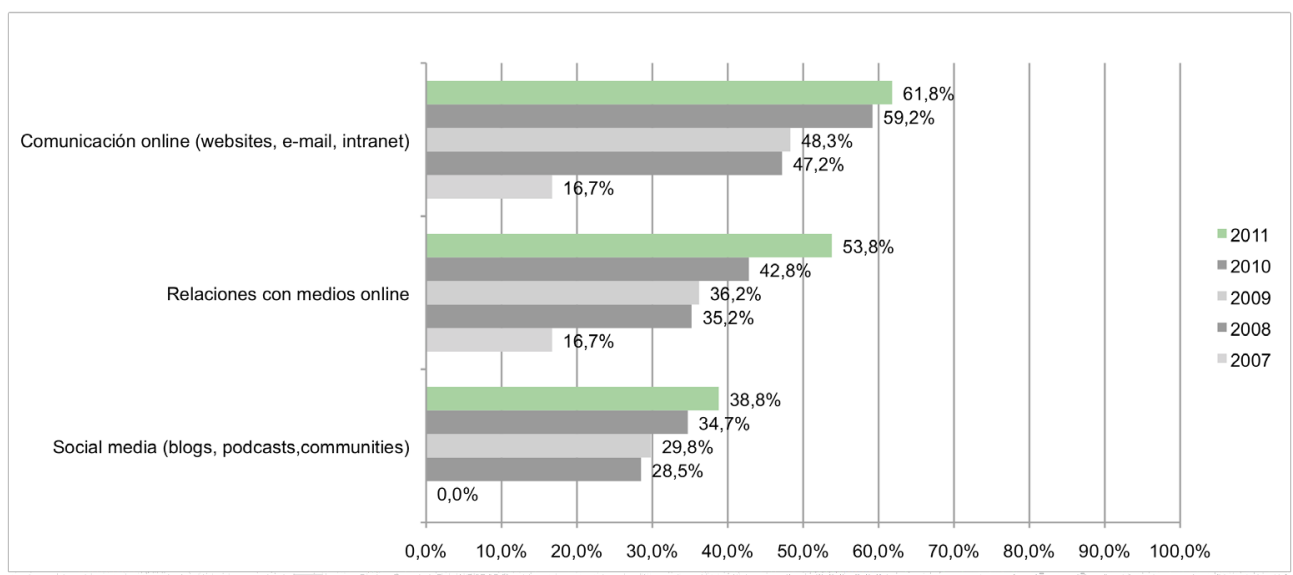

Fuente: elaboración propia. 
Por otra parte, el análisis longitudinal permite afirmar que las previsiones realizadas por los profesionales españoles sobre el desarrollo de los diferentes canales no suelen cumplirse, especialmente en los aspectos relacionados con la evolución de los medios tradicionales y con el excesivo optimismo del sector sobre la introducción de las nuevas herramientas digitales. Aunque en 2008 los encuestados pronosticaron una caída del 21,8 por ciento en las relaciones con los medios impresos de cara al año 2011, la realidad fue que el recorte se limitó a tan solo 4,7 puntos. Además, el 26,1\% de incremento previsto para la comunicación online se tradujo en un 14,6\% y el $21,1 \%$ esperado para los Social Media, finalmente se convirtió en una subida del 10,3\%.
Es decir, como se aprecia en la gráfico 5, los encuestados sobrevaloraron el desarrollo de la mayor parte de los canales (comunicación online, cara a cara, social media, relaciones con los medios online, eventos y sponsorización) pero erraron al predecir una caída de los medios tradicionales, tanto impresos como audiovisuales, y de las publicaciones corporativas. (Gráfica 5)

\section{Los 'Social Media' y sus herramientas de comunicación}

El término 'Social Media' engloba una gran variedad de herramientas de comunicación que a lo largo de los últimos años están evolucionando de forma desigual en el marco de la estrategia de

Gráfica 5. Predicción de evaluación de los canales de comunicación (2008-2011)

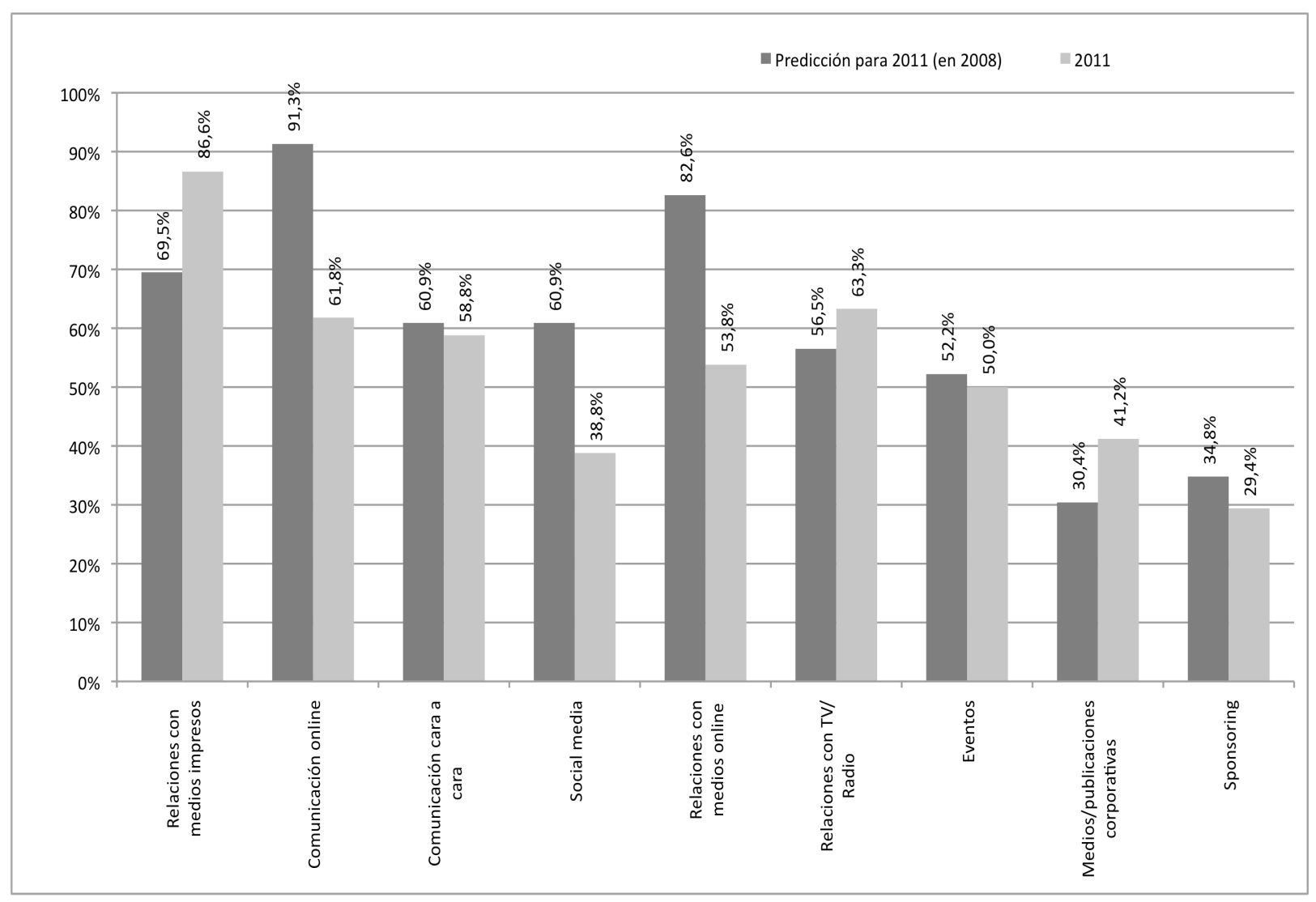

Fuente: elaboración propia. 
comunicación de las organizaciones españolas. El éxito inusitado de las redes sociales se pone de manifiesto con un crecimiento cercano al 30\% en los últimos cuatro años, situándose a la cabeza del arsenal de herramientas colaborativas que están contribuyendo a fomentar el diálogo con los grupos de interés. Los vídeos online también han ido ganando terreno ( $+21 \%)$, aunque todavía se mantienen por detrás de los weblogs, que ocupan el segundo lugar en la lista de canales más aprecidados por parte de los profesionales. El microblogging (Twitter) se puede considerar como un fenómeno de reciente incorporación -en 2008 no se incluyó en el cuestionario- pero en tan solo tres años es valorado positivamente por el 39,1\% de los encuestados y se espera que su influencia continúe aumentando.

Sin embargo, encontramos tres herramientas con mayor trayectoria que las redes sociales o el microblogging, pero que no acaban de despe- gar en el ámbito de las relaciones públicas: los podcasts y los mundos virtuales, que en cuatro años solo han crecido un $1,5 \%$ en importancia, y los wikis, con un ligero aumento del 2,9\%. (Gráfica 6)

\section{Evolución de las características de los profesionales}

La mayor parte de los encuestados lleva más de diez años ejerciendo la profesión, aunque la veteranía se ha reducido ligeramente en los últimos cinco años $(-4,4 \%)$. El colectivo que más ha crecido es el intermedio, es decir, el de los relaciones públicas con una trayectoria que osciala entre los 6 y los diez años (+10,8\%). En definitiva, no se ha producido un cambio significativo del perfil de los profesionales españoles en lo que respecta a la experiencia laboral ya que los cambios que se aprecian son mínimos. (Gráfica 7)

Gráfica 6. Desarrollo de los Social Media

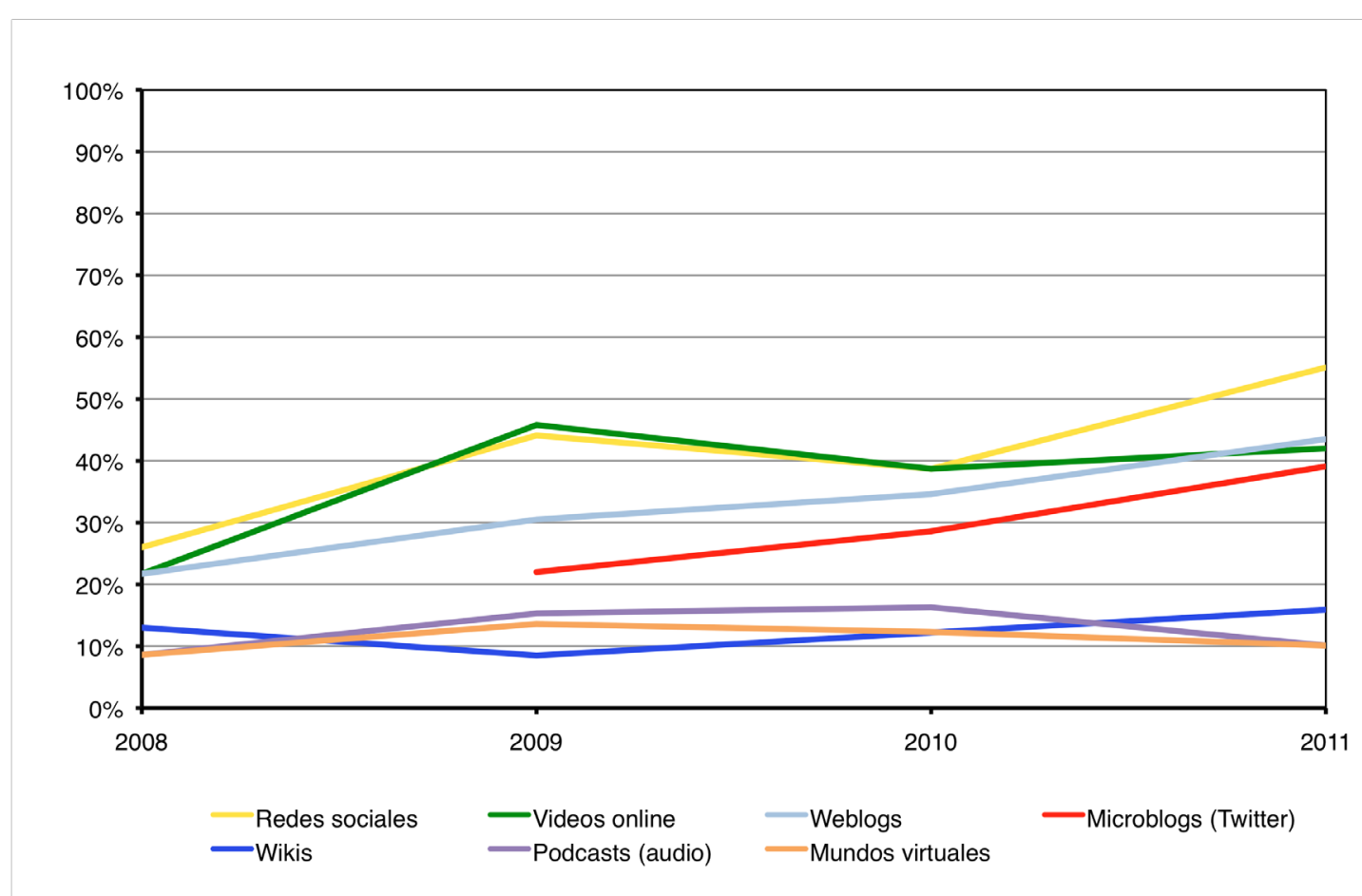

Fuente: elaboración propia. 
Gráfica 7. Experiencia laboral

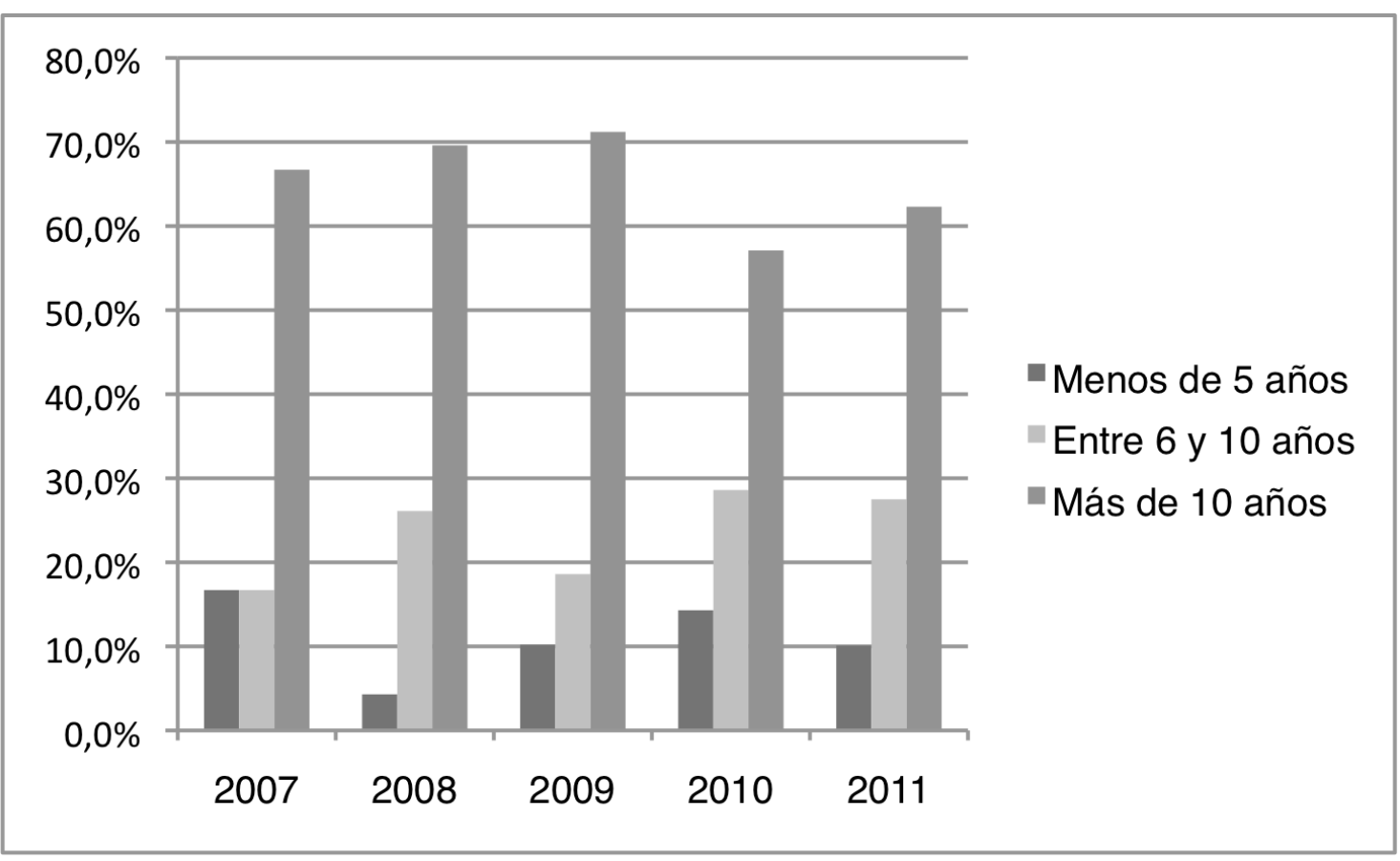

Fuente: elaboración propia.

El salario más habitual de los profesionales de la comunicación en España en 2009 se situaba entre los 60.000 y los 10.000 euros (37\%), mientras en 2010 y 2011, la franja más numerosa la ocupaban los trabajadores que cobraban entre $30.000 \mathrm{y}$ 60.000 euros (40,8\% y $41,6 \%$, respectivamente) $)^{1}$. Este dato demuestra una tendencia hacia la polarización en los ingresos ya que en estos últimos tres años también crecen los encuestados que declaran cobrar más de 150.000 euros. (Gráfica 8)

En cuanto a la formación acreditada por los profesionales españoles, no se observa un cambio de tendencia entre 2009 y 2011 , destacando el número de titulados en comunicación, seguidos de lejos por los ofrecen una certificación profesional en Relaciones Públicas o cualquier otra disciplina de comunicación. (Tabla 1)

1 Algunos de los datos sociodemográficos que se presentan en este apartado no pueden ofrecerse de manera comparada para los años 2007 y 2008.
Por otra parte, se aprecia un descenso del número de profesionales de las relaciones públicas y gestión de la comunicación que trabajan en agencias de comunicación $(-8,7 \%)$ y en empresas que cotizan en bolsa $(-2,9 \%)$, al tiempo que se ha producido un incremento de trabajadores en empresas no cotizadas $(+8,5 \%)$, menos apreciable en el caso de las organizaciones gubernamentales $(+1,5)$ y las organizaciones sin ánimo de lucro $(+1,4 \%)$. (Gráfica 9)

\section{Conclusiones}

El análisis longitudinal de los resultatos obtenidos a través del European Communication Monitor (ECM) entre los años 2007 y 2011 revela la importancia que otorgan los profesionales españoles de las relaciones públicas a la capacidad para manejar la evolución digital y los medios sociales, una tendencia que también se observa en el rápido crecimiento de los canales de comunicación online. Respecto a los temas 
Gráfica 8. Salarios

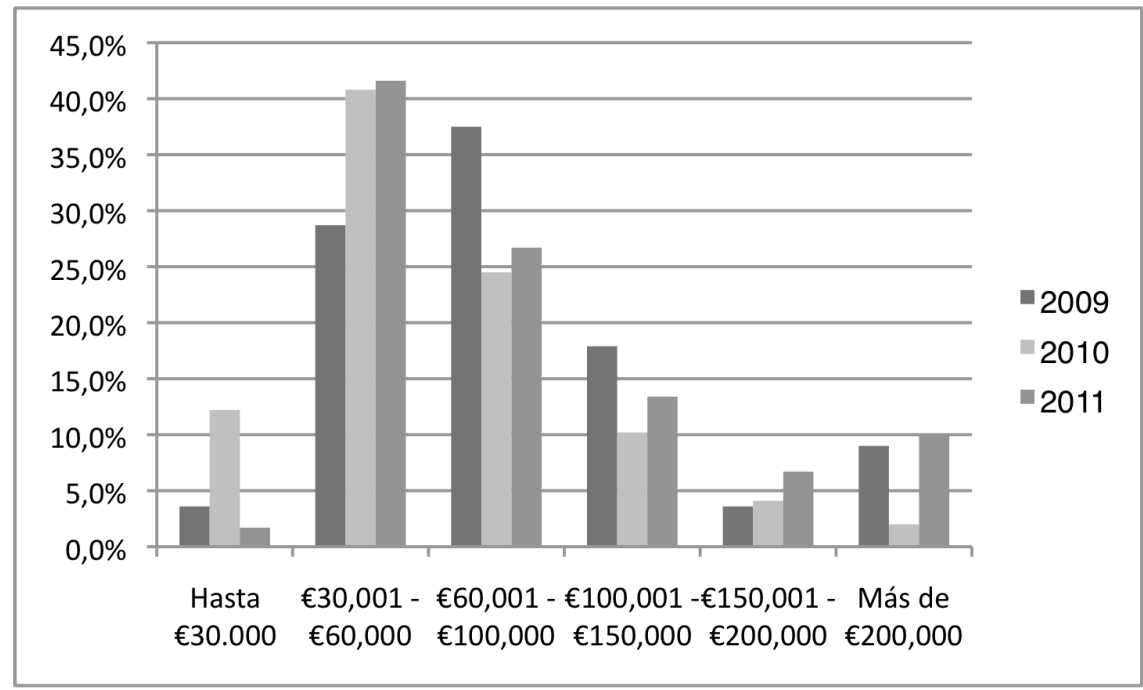

Fuente: elaboración propia.

Tabla 1. Formación académica (2009-2011)

\begin{tabular}{|l|c|c|c|}
\hline & 2009 & 2010 & 2011 \\
\hline Grado en comunicación (Licenciado/Máster/Doctorado) & $64,4 \%$ & $61,2 \%$ & $65,2 \%$ \\
\hline Certificado profesional en PR/Comunicación & $20,3 \%$ & $18,4 \%$ & $18,2 \%$ \\
\hline Certificado profesional en otra disciplina de comunicación & $20,3 \%$ & $24,5 \%$ & $20,3 \%$ \\
\hline
\end{tabular}

Gráfica 9. Organizaciones en las que trabajan

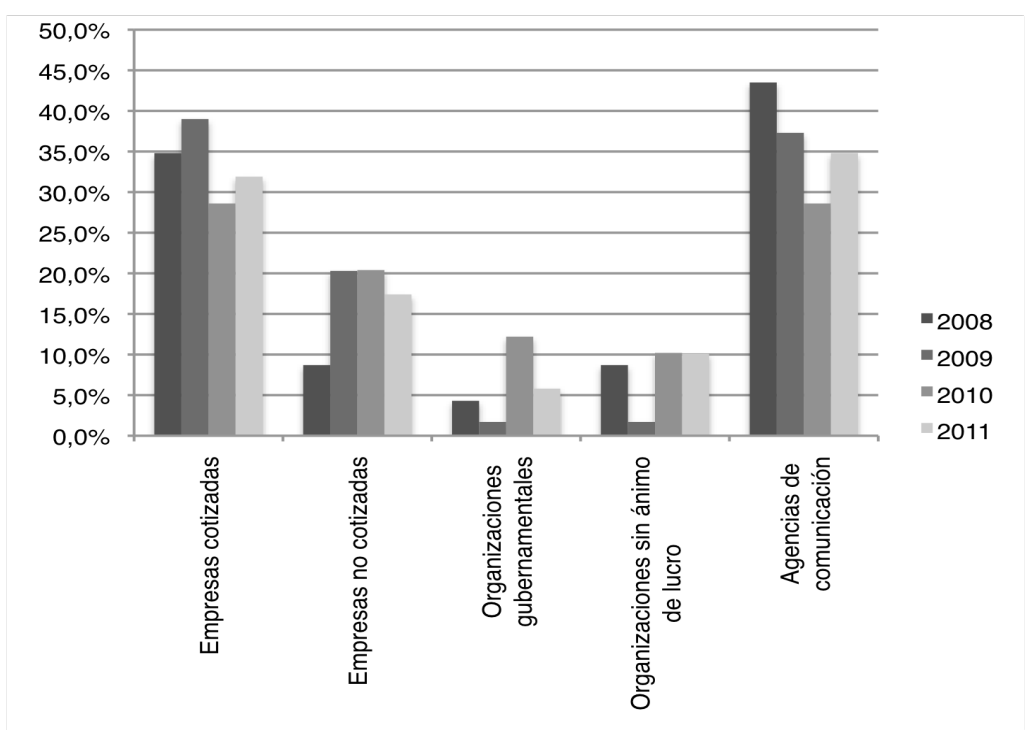

Fuente: elaboración propia. 
estratégicos para el sector, también se aprecia el peso que va adquiriendo la evaluación y la necesidad de establecer nuevos métodos para evaluar la comunicación, además de los retos que están surgiendo a raíz de la globalización.

Respecto a las disciplinas más relevantes, la comunicación corporativa no solo se mantiene en el lugar más destacado, sino que, además, ha crecido en los últimos cinco años, al igual que la comunicación de marketing, que ocupa el segundo lugar en importancia para los trabajadores del sector de las relaciones públicas. Se aprecia, sin embargo, una caída de la gestión de los asuntos públicos y de la comunicación de crisis.

Las previsiones de los encuestados no se cumplen y las relaciones con los medios impresos siguen siendo el instrumento más valorado, demostrando una fortaleza por la que muy pocos apostaban en años anteriores. El claro incremento de la comunicación online no amenaza su hegemonía, al menos por el momento, aunque sí el segundo lugar que ocupan las relaciones con la radio y la televisión. Los Social Media crecen, arrastrados sobre todo por las redes sociales, los vídeos online y los weblogs y los microblogs (Twitter), que se han convertido en las herramientas más atractivas para los responsables de gestionar la comunicación en las organizaciones. Los podcasts, los wikis y los mundos virtuales, sin embargo, no consiguen depegar y se mantienen a niveles similares de 2007.

En cuanto a las características sociodemográficas de los profesionales españoles, aunque no se aprecian cambios significativos, sí destaca un ligero incremento del número de profesionales con una experiencia laboral que oscila entre los 6 y los 10 años, mientras disminuyen los veteranos. Por último, suben los trabajadores en empresas no cotizadas, organizaciones no gubernamentales y sin ánimo de lucro, al tiempo que se reducen en las empresas que cotizan en bolsa y las agencia de comunicación.

\section{Referencias bibliográficas}

ADECEC. (2002). La comunicación y las relaciones públicas en España. Radiografía de un sector. Madrid: Pirámide.

ADECEC. (2004). La comunicación y las relaciones públicas en España. Radiografía de un sector. Madrid. Recuperado el 7 de enero de 2012 de, http://www.adecec.com.

ADECEC. (2008). La comunicación y las relaciones públicas en España. Radiografía de un sector. Madrid. Recuperado el 7 de enero de 2012 de, http://www.adecec.com.

Arceo Vacas, J. L. (2003). Perfil de los profesionales de la comunicación persuasiva en España. Madrid: ICIE, Universidad Complutense.

Arceo Vacas, J. L. (coord.) (2004). Las Relaciones Públicas en España. Madrid: McGraw-Hill.

Beurer-Züllig, B., Fieseler, C., \& Meckel, M. (2009). A descriptive inquiry into the corporate communication profession in Europe. Public Relations Review, 35(3), 270-279.

Castillo, A. (2004). Investigación sobre la evolución histórica de las relaciones públicas. Historia y Comunicación Social, 9, 43-62.

Coombs, W. T., Holladay, S., Hasenauer, G., ySignitzer, B. (1994). A comparative analysis of international public relations: Identification and interpretation of similarities and differences between professionalization in Austria, Norway, and the United States. Journal of Public Relations Research, 6, pp. 23-39.

Culbertson, H. M., E Chen, N. (1996). International public relations. a comparative analysis. Mahwah, New Jersey: Lawrence Erlbaum Associates.

De Santo, B., E Moss, D. (2004). Rediscovering what PR managers do: rethinking the measurement of managerial behaviour in the public relations context. Journal of Communication Management, 9(2), pp. 179-196.

Dircom (2000). El estado de la comunicación en España: empresas y organismos públicos e instituciones. Madrid: Dircom. 
Dircom (2005). El estado de la Comunicación en España. Madrid: Dircom.

Dircom (2010). El estado de la Comunicación en España. Madrid: Dircom.

Fernández, J. y Huertas, A. (2005). Análsis del sector de las Relaciones Públicas en Tarragona. La otra realidad de la profesión en España. Actas del II Congreso Internacional de Investigación y Relaciones Públicas. Universidad de Sevilla.

Grunig, J. E., Grunig, L.A., Sriramesh, K., Huang, Y. H., E Lyra, A. (1995). Models of public relations in an international setting. Journal of Public Relations Research, 7, pp. 163-186.

Gutiérrez-García, E. (2010). Gobierno corporativo y comunicación empresarial. ¿Qué papel cumplen los directores de comunicación en España? Palabra Clave, 3(1), pp 147-160.

Jang, H. (1997). Cultural differences in an interorganizational network: shared public relations firms among Japanese and American companies. Public Relations Review, 23, pp. 341-379.

Kent, M. L. E Taylor, M. (2007). Beyond excellence: Extending the generic approach to international public relations. The case of Bosnia. Public Relations Review, 33 (1), pp. 10-20.

Lim, S., Goh, J., E Sriramesh, K. (2005). Applicability of the generic principles of excellent public relations in a different cultural context. The case study if Singapore, Journal of Public Relations Research, 17, pp. 315-340.

Matilla, K. y Marca, G. (2011). La función estratégica del Dircom en España en 2010. Revista Internacional de Relaciones Públicas, 2 (1), pp. 11-23.

Míguez, M. I. (2007). Aproximación a la investigación y a la situación de las relaciones públicas en Europa. Estudio comparativo entre el caso español y el alemán. Revista Latina de Comunicación Social, 62. Recuperado el 22 de febrero de 2012, de http://www.ull.es/publicaciones/latina/200715 Miguez.htm
Moreno, M. A. (2004). «Spain». En: B. Van Ruler; D. Verčič (ed.). Public Relations and Communication Management in Europe. Berlín: Mouton de Gruyter, p. 393- 412.

Moreno, A., Molleda, J.C. y Suárez, A.M. (2007). Comunicación estratégica y relaciones públicas en entornos socioeconómicos y políticos en transición: estudio comparativo en Colombia, México y Venezuela. Razón y Palabra, 51.

Moreno, Á., Verhoeven, P., Tench, R., Zerfass, A., E Zerfass, A. (2010). European Communication Monitor 2009. El desarrollo de la profesión de relaciones públicas y gestión de la comunicación estratégica en Europa. Razón y Palabra, 72.

Moreno, Á., Zerfass, A. y Navarro, C. (2010). La situación de los profesionales de relaciones públicas y comunicación estratégica en Europa y en España. Análisis comparado del European Communication Monitor 2009. Actas Congreso AIPR. Barcelona.

Moreno, Á., Zerfass, A. y Navarro, C. (2011). European Communication Monitor 2010: España en línea con los profesionales europeos. En: VV.AA. Anuario de la Comunicación 2011. Madrid: Dircom.

Nessmann, K. (1995). Public relations in Europe: a comparison with the United States. Public Relations Review, 21(2), pp. 151-160.

Noguero, A. (2004). Principales acontecimientos en la evolución de las Relaciones Públicas en España. En: J.L. Arceo (dir.). Las Relaciones Públicas en España. Madrid: McGraw-Hill, pp. 337-359.

O'Connor, N., E Muzi, T. (2004). Profiling the regulatory environment of public relations practice in the UK, Italy, and South Africa. Journal of Communication Management, 9 (1), pp.28-56.

Sriramesh, K. E Kim, Y. (1999). Public relations in three Asian cultures: an análisis. Journal of Public Relations Research, 11(4), pp. 271-292.

Sriramesh, K. E Verčič, D. (2003). A theoretical framework for global public relations research and practice. In K. Sriramesh y D. Verčič (Eds.), The global public relations handbook; theory, research, and practice. 
New Jersey, NJ: Lawrence Erlbaum Associates, pp. $1-19$.

Swerling, J., Gregory, J., Schuh, J., Goff, T., Gould, J, Gu, X. C., Palmer, K., y Mchargue, A. (2008). Fifth Annnual Public Relations Generally Accepted Practices (G.A.P.) Study. GAP V. Los Angeles: University of Southern California. Available at: http://annenberg. usc.edu/CentersandPrograms/ResearchCenters/ SPRC.aspx

Swerling, J., Mitroff, I., Floto, J., Hall, J. y Bishop, G. (2003). Second Annnual Public Relations Generally Accepted Practices (GAP) Study GAP II. Los Angeles: University of Southern California. Available at: http:// annenberg.usc.edu/CentersandPrograms/ResearchCenters/SPRC/PrevGAP.aspx

Swerling, J., Mitroff, I., Hall, J., King, D., Benson, L. y ODBoyle, P. (2005). Forth Annnual Public Relations Generally Accepted Practices (G.A.P.) Study GAP IV. Los Angeles: University of Southern California. Available at: http://annenberg.usc.edu/CentersandPrograms/ResearchCenters/SPRC/PrevGAP.aspx

Swerling, J., Mitroff, I., Hall, J., King, D., Zeltzer, D. y Waldman, T. (2004). Tird Annnual Public Relations Generally Accepted Practices (G.A.P.) Study GAP III. Los Angeles: University of Southern California. Available at: http://annenberg.usc.edu/CentersandPrograms/ ResearchCenters/SPRC/PrevGAP.aspx

Tilson, D.J. y Alozie, E.C. (2004). Toward the common good; perspectives in international public relations. Boston, MA: Allyn and Bacon.

Tison, D. y Saura, P. (2003). Public Relations and the New Golden Age of Spain: A Confluence of Democracy, Economic Development and the Media. Public Relations Review, 29 (2), pp. 125-143.

Verčič, D.; Grunig, L.A., y Grunig, J.E. (1996). Global and specific principles of public relations: evidence from Slovenia. In H.M. Culbertson y N. Chen (Eds.), International public relations; a comparative análisis. New Jersey, NJ: Lawrence Erlbaum Associates, pp. 31-66.

Verhoeven, P. E Aarts, N. (2010). How European public relations men and women perceive the impact of their professional activities. PRism 7(4): http://www. prismjournal.org.

Xifra Triadú, Jordi (2008): Modelos de las relaciones públicas políticas: Análisis de la situación en Cataluña. Revista Latina de Comunicación Social, 63, pp. 392-399. La Laguna (Tenerife): Universidad de La Laguna, recuperado el 23 de diciembre de 2011, de http:// www.revistalatinacs.org/08/32_ $789 \quad 50$ _Girona/ Jordi Xifra.html DOI: 10.4185/RLCS-63-2008-789392-399

Zerfass, A., Van Ruler, B., Rogojinaru, A., Verčič, D., y Hamrefors, S. (2007). European Communication Monitor 2007. Trends in Communication Management and Public Relations-Results and Implications. Leipzig: University of Leipzig/Euprera. Available at: www.communicationmonitor.eu

Zerfass, A., Moreno, A., Tench, R., Verčič, D., y Verhoeven, P. (2008). European Communication Monitor 2008. Trends in Communication Management and Public Relations-Results and Implications. Brussels, Leipzig: Euprera/University of Leipzig Available at: www.communicationmonitor.eu

Zerfass, A., Moreno, A., Tench, R., Verčič, D., y Verhoeven, P. (2009). European Communication Monitor 2008. Trends in Communication Management and Public Relations. Results of a survey in 34 countries. Brussels: EACD, EUPRERA.

Zerfass, A., Tench, R., Verhoeven, P., Verčič, D., \& Moreno, A. (2010):European Communication Monitor 2010. Status Quo and Challenges for Public Relations in Europe. Results of an Empirical Survey in 46 Countries (Chart Version). Brussels: EACD, EUPRERA (available at: www.communicationmonitor.eu)

Zerfass, A., Verhoeven, P., Tench, R., Moreno, A., E Verčič, D. (2011): European Communication Monitor 2011. Empirical Insights into Strategic Communication in Europe. Results of an Empirical Survey in 43 Countries (Chart Version). Brussels: EACD, EUPRERA (available at: www.communicationmonitor.eu) 